\title{
Validated Spectrophotometric Methods for Simultaneous Determination of Food Colorants and Sweeteners
}

\author{
Fatma Turak and Mahmure Ustun Ozgur \\ Department of Chemistry, Faculty of Science and Art, Yildiz Technical University, 34220 Istanbul, Turkey \\ Correspondence should be addressed to Mahmure Ustun Ozgur; mozgur@yildiz.edu.tr
}

Received 30 November 2012; Accepted 14 January 2013

Academic Editor: Ghada M. Hadad

Copyright (C) 2013 F. Turak and M. U. Ozgur. This is an open access article distributed under the Creative Commons Attribution License, which permits unrestricted use, distribution, and reproduction in any medium, provided the original work is properly cited.

\begin{abstract}
Two simple spectrophotometric methods have been proposed for simultaneous determination of two colorants (Indigotin and Brilliant Blue) and two sweeteners (Acesulfame-K and Aspartame) in synthetic mixtures and chewing gums without any prior separation or purification. The first method, derivative spectrophotometry (ZCDS), is based on recording the first derivative curves (for Indigotin, Brillant Blue, and Acesulfame-K) and third-derivative curve (for Aspartame) and determining each component using the zero-crossing technique. The other method, ratio derivative spectrophotometry (RDS), depends on application ratio spectra of first- and third-derivative spectrophotometry to resolve the interference due to spectral overlapping. Both colorants and sweeteners showed good linearity, with regression coefficients of $0.9992-0.9999$. The LOD and LOQ values ranged from 0.05 to $0.33 \mu \mathrm{gmL}^{-1}$ and from 0.06 to $0.47 \mu \mathrm{gmL}^{-1}$, respectively. The intraday and interday precision tests produced good RSD $\%$ values $(<0.81 \%)$; recoveries ranged from $99.78 \%$ to $100.67 \%$ for all two methods. The accuracy and precision of the methods have been determined, and the methods have been validated by analyzing synthetic mixtures containing colorants and sweeteners. Two methods were applied for the above combination, and satisfactory results were obtained. The results obtained by applying the ZCDS method were statistically compared with those obtained by the RDS method.
\end{abstract}

\section{Introduction}

Colorants are added to foods to make them more attractive, replacing their natural color that can be lost during the industrial process or to avoid variations in the color of the final product. The trouble is that some synthetic azo dyes can be toxic to the human health and when in contact with some drugs can cause allergic and asthmatic reactions to some people, induced the development of cancer and others diseases [1]. In this way, in the last years, efforts have been made to control and to limit the amount of synthetic colorants that are added in foods, whereas the more toxic dyes have been banned. Thus, it is necessary to have efficient methodologies to control the amount of colorants in foods. The synthetic indigotin dye, indigotin (indigo carmine, E 132, IND), and the synthetic azo dye, brilliant blue (E 133, BB) are among the colorants used in common foods such as sweets, drinks, ice cream, and chewing gum. As with many other food additives, the analytical control of these colorants is of considerable importance in the food industry because of their toxic and carcinogenic potential $[2,3]$. Several methods have been proposed for the codetermination of colorants in mixtures. These methods include UV/VIS spectrophotometry [4-8], chromatography [1,9-12], capillary electrophoresis [13], differential pulse polarography [14], voltammetry [15], and chemometric techniques [16-19].

Artificial sweeteners are also widely used in food, beverage, confectionary, and pharmaceutical industries throughout the world. They are the modern non-caloric alternatives to sugars as additives in foods and drinks. Consumers select low-calorie foods added with artificial sweeteners to decrease or to control calorie intake and thus body mass and to aid control of certain health or medical conditions such as diabetes and hypoglycemia. The acceptable daily intake (ADI) values, determined by Joint FAO/WHO Expert Committee on Food Additives, are $0-40 \mathrm{mg} \mathrm{Kg}^{-1}$ body mass for aspartame (ASP, E 951) and $0-15 \mathrm{mg} \mathrm{Kg}^{-1}$ for acesulfameK(ACE-K, E 950) [20]. The determination of ACE-K and 
ASP in samples with the other sweeteners or additives has been carried out by high performance liquid chromatography (HPLC) [21-24]. In addition, ion chromatography (IC) offers an attractive alternative to traditional HPLC methods [25]. In the past few years, micellar electrokinetic chromatography (MEKC) and capillary zone electrophoresis (CZE) have been applied to the simultaneous determination of several kinds of sweeteners [26]. Other methods less commonly used for the Acesulfame- $\mathrm{K}$ and Aspartame determination are Fourier transform Raman spectrometry [27], chemometry [28-30], and the others [31] (Scheme 1).

Chromatography and capillary electrophoresis are very suitable when the sample contains several colorants or sweeteners, and these methods need special equipment that may not be available in certain quality laboratories. All multivariate calibration methods, including partial least squares (PLS), require the data processing with powerful software as well as the manipulations of the abstract vector space, and its application to the regression analysis and various chemometric methods are often used for more complex mixtures. But the foods, beverages, and pharmaceuticals contain only one, two, or rarely three colorants and sweeteners. Thus, analytical methods for alternative technique are always useful, especially if the methods are simple, cheap, and comparatively fast. Spectrophotometry, as an alternative methodology, is suitable for routine laboratories especially for developing countries, and sometimes, serious analytical problems can be resolved by this common technique. One of the classic analytical problems of spectrophotometric multicomponent analysis is that the analyte of interest is often accompanied by other compounds absorbing in the same spectral region. Under computer-controlled instrumentation, derivative spectrophotometry is playing a very important role in the resolution of band overlapping in quantitative analysis [32]. However, for binary mixtures, the classical zero-crossing method requires often to use a wavelength with low sensitivity in the measurements. Salinas et al. [33] designed another spectrophotometric method, which is based on the derivation of the ratio-spectra to resolve binary mixtures. The main advantage of the ratio derivative spectrophotometry is the chance of performing easy measurements in correspondence of peaks so it permits the use of the wavelength of highest value of analytical signals (a maximum or a minimum), and moreover, the presence of a lot of maxima and minima is another advantage by the fact that these wavelengths give an opportunity for the determination of active compounds in the presence of other compounds and ingredients which possibly interfere with the assay. Although a great variety of methods have been applied to the analysis of the aforementioned compounds in foods, there is no report about simultaneous estimation of this combination in synthetic mixtures or in food samples.

The aim of this work is to develop easy, sensitive, and fast spectrophotometric methods that can be applied for the routine analysis of the colorants (BB and IND) and sweeteners (ASP and ACE-K) simultaneously in the quaternary laboratory mixtures, foods, and drinks without previous separation. In the present study, measurements were assayed zero-crossing and ratio spectra derivative techniques
[34-36]. Two methods were successfully applied for the above combination, and satisfactory results were obtained. No extraction, no evaporation step, no complexation agent, and no harmful chemicals are involved in the suggested methods, in that connection decreasing time and the error in quantitation and therefore can be used for routine analysis of both colorants in quality control and routine laboratories.

\section{Material and Methods}

2.1. Instruments. A double-beam Shimadzu 2450 UV-VIS spectrophotometer, connected to personal computer compatible with a laser printer, was used. The bundle software, version 2.21, was used to process the absorption and derivative spectra. The spectral band width was $1 \mathrm{~nm}$ and scanning speed was medium. Magnetic stirrer (Arex-Velp Scientifica), Hettich EBA 20 centrifuge, and $0.45 \mu \mathrm{m}$ membranes were used in this study.

2.2. Reagents and Materials. All chemicals were analytical grade, and double distilled water was used throughout the experiments. The standard synthetic colorants were Brilliant Blue (CI 42090, CAS 3844-45-9) from SigmaAldrich (Steinheim, Germany) and Indigotin (CI 73015, CAS 482-89-3) from Sigma-Aldrich (Steinheim, Germany). The standard sweeteners were Acesulfame-K (CAS 55589-62-3) from Sigma-Aldrich (Steinheim, Germany) and Aspartame (CAS 22839-47-0). Chewing-gum samples were purchased from local markets. The commercial chewing-gum samples, containing gum base, sweeteners (E950, E951), softeners, bulking agents, flavorings, colorants (E132, E133), preservatives, moisture trap, acid regulating, and antioxidant (BHA), were studied. The ingredients are in order decreasing of concentration.

2.3. Solutions. Stock solutions $\left(100 \mu \mathrm{gmL}^{-1}\right)$ of ASP and ACE-K were prepared separately by dissolving appropriate weights of pure product in distilled water. These solutions were freshly prepared and protected from light. Stock solutions of BB and IND $\left(100 \mu \mathrm{gmL}^{-1}\right)$ were also prepared in distilled water. These stock solutions were further diluted with distilled water to obtain working standard solutions of suitable concentrations (corresponding to the linearity range stated in Table 1). Accurate volumes of each of ASP, ACE$\mathrm{K}, \mathrm{BB}$, and IND stock solution were transferred into $10 \mathrm{~mL}$ volumetric flasks and diluted to volume with distilled water to prepare synthetic mixtures within the concentration range (2-10 $\mathrm{gmL}^{-1}$ ) of each compound (Table 2).

\subsection{Procedures}

\subsubsection{Spectrophotometric Measurements}

(a) Sample Preparation. 50 chewing-gum samples were weighed and cut into small pieces. An accurately weighed sample equivalent to ten chewing-gums was taken to erlenmeyer flask. $35 \mathrm{~mL}$ of water was added and stirred with 


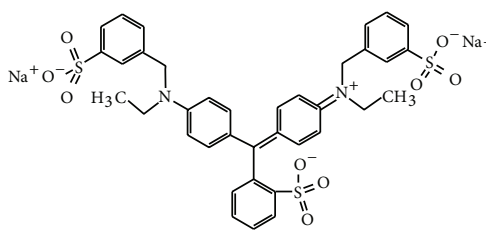

Brillant Blue

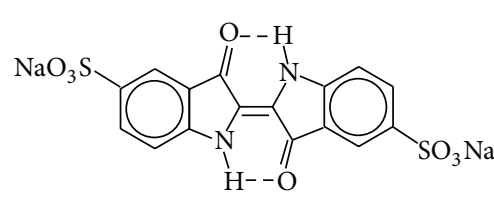

Indigotin<smiles>[Y]N1[O+]OC(C)=CC1=O</smiles>

Acesulfame-K

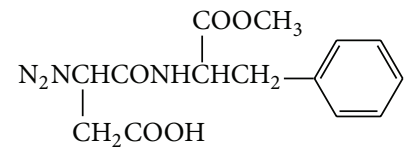

Aspartame

Scheme 1: Molecular structures of Brilliant Blue (E133) Indigotin (E132), Acesulfame-K (E950), and Aspartame (E951).

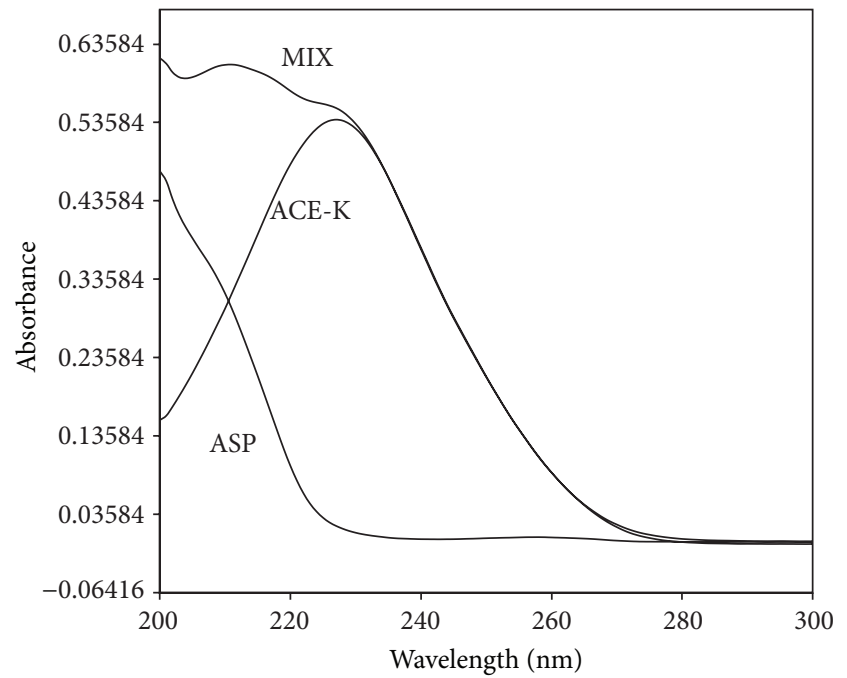

(a)

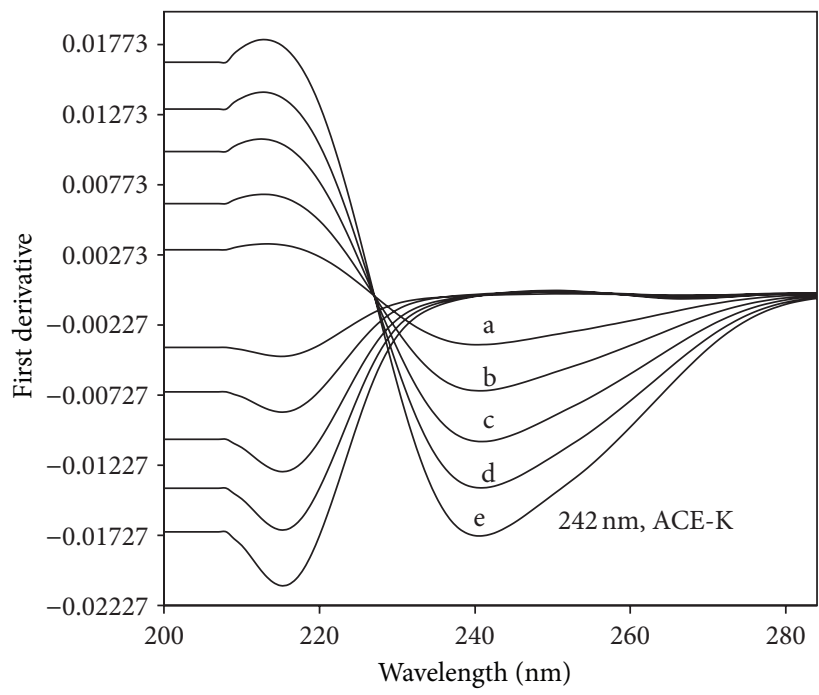

(b)

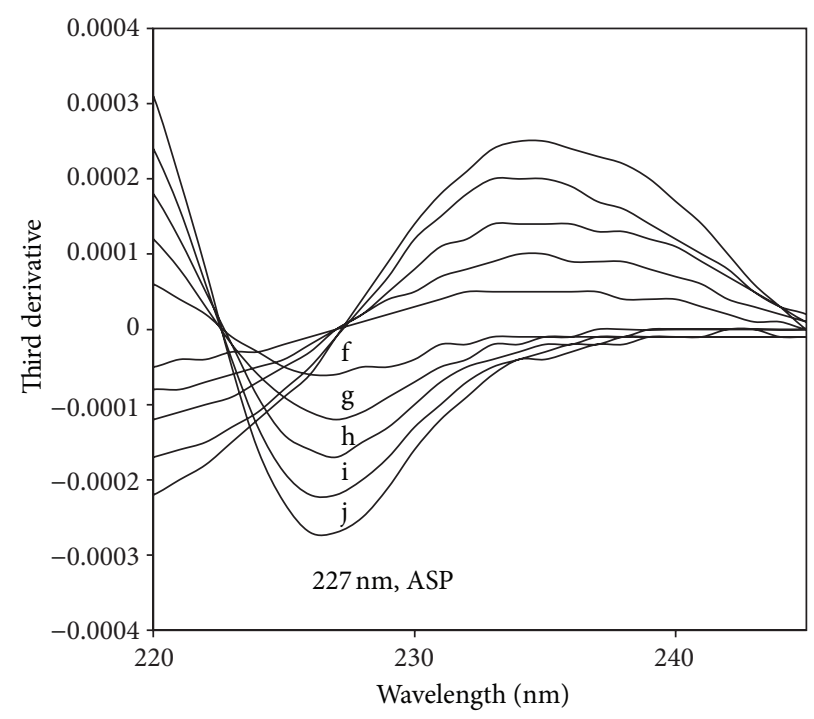

(c)

FIGURE 1: Zero-order spectra (a) of $10 \mu \mathrm{g} \cdot \mathrm{mL}^{-1}$ ASP, $10 \mu \mathrm{g} \cdot \mathrm{mL}^{-1}$ ACE-K, and their binary mixture, first-derivative spectra (b), and thirdderivative spectra (c) of sweeteners (ACE-K: a: 2, b: 4, c: 6, d: 8, e: $10 \mu \mathrm{gmL}^{-1}$; ASP: f: 2, g: 4, h: 6, i: 8, j: $10 \mu \mathrm{gmL}^{-1}$ ). 
TABLE 1: Statistical parameters of the simultaneous determination of colorants (IND, BB) and sweeteners (ACE-K, ASP) by ZCDS and RDS methods.

\begin{tabular}{lccccccc}
\hline Methods & Analyte & $\begin{array}{c}\text { Selected } \\
\text { Wavelength } \\
(\mathrm{nm})\end{array}$ & $\begin{array}{c}\text { Concentration } \\
\text { range } \\
\left(\mu \mathrm{gmL}{ }^{-1}\right)\end{array}$ & Regression equations ${ }^{\mathrm{a}}$ & $\begin{array}{c}\text { Correlation } \\
\text { coefficient } \\
(r)\end{array}$ & $\begin{array}{c}\text { Detection } \\
\text { Limit } \\
\left(\mu \mathrm{gmL}^{-1}\right)\end{array}$ & $\begin{array}{c}\text { Quantification } \\
\text { Limit } \\
\left(\mu \mathrm{gmL}^{-1}\right)\end{array}$ \\
\hline \multirow{6}{*}{ ZCDS } & $\mathrm{IND}$ & ${ }^{1} \mathrm{D}_{348}$ & $2-10$ & $4.53 \cdot 10^{-4} \mathrm{C}^{*}-1.24 \cdot 10^{-4}$ & 0.9998 & 0.33 & 0.47 \\
& $\mathrm{BB}$ & ${ }^{1} \mathrm{D}_{420}$ & $2-10$ & $6.39 \cdot 10^{-4} \mathrm{C}^{*}-6.2 \cdot 10^{-5}$ & 0.9999 & 0.18 & 0.34 \\
& $\mathrm{ACE}-\mathrm{K}$ & ${ }^{1} \mathrm{D}_{242}$ & $2-10$ & $1.75 \cdot 10^{-3} \mathrm{C}^{*}$ & 0.9999 & 0.04 & 0.10 \\
\hline \multirow{4}{*}{$\mathrm{RDS}$} & $\mathrm{ASP}$ & ${ }^{3} \mathrm{D}_{227}$ & $2-10$ & $2.75 \cdot 10^{-5} \mathrm{C}^{*}-3 \cdot 10^{-6}$ & 0.9995 & 0.18 & 0.33 \\
& $\mathrm{IND}$ & ${ }^{1} \mathrm{D}_{356}$ & $2-10$ & $0.074 \mathrm{C}^{*}+4.7 \cdot 10^{-3}$ & 0.9994 & 0.44 \\
& $\mathrm{BB}$ & ${ }^{1} \mathrm{D}_{419}$ & $2-10$ & $0.055 \mathrm{C}^{*}+6.95 \cdot 10^{-3}$ & 0.9992 & 0.14 & 0.22 \\
& $\mathrm{ACE}-\mathrm{K}$ & ${ }^{1} \mathrm{D}_{248}$ & $2-10$ & $1.01 \mathrm{C}^{*}+0.071$ & 0.9998 & 0.07 & 0.08 \\
& $\mathrm{ASP}$ & ${ }^{3} \mathrm{D}_{225}$ & $2-10$ & $2.02 \cdot 10^{-5} \mathrm{C}^{*}-1 \cdot 10^{-6}$ & 0.9999 & 0.05 & 0.06 \\
\hline
\end{tabular}

Five separate determinations were performed and mean calculated.

${ }^{*} \mathrm{C}$ is the concentration of the analyte $\left(\mu \mathrm{g} \mathrm{mL} L^{-1}\right)$.

TABLE 2: Method validation for the determination of laboratory prepared mixtures of IND, BB, ACE-K and ASP by the proposed methods.

\begin{tabular}{|c|c|c|c|}
\hline Parameter & Accuracy mean ${ }^{*} \pm \mathrm{RSD} \%$ & Precision repeatability $^{\mathrm{a}} \pm \mathrm{RSD} \%$ & Intermediate precision $^{\mathrm{b}} \pm \mathrm{RSD} \%$ \\
\hline \multicolumn{4}{|l|}{ ZCDS } \\
\hline $\operatorname{IND}\left({ }^{1} \mathrm{D}_{348}\right)$ & $100.76 \pm 0.77$ & $99.95 \pm 0.58$ & $100.28 \pm 0.24$ \\
\hline $\mathrm{BB}\left({ }^{1} \mathrm{D}_{420}\right)$ & $99.96 \pm 0.73$ & $100.68 \pm 0.58$ & $99.86 \pm 0.31$ \\
\hline ACE-K $\left({ }^{1} D_{242}\right)$ & $100.65 \pm 0.49$ & $100.05 \pm 0.09$ & $100.09 \pm 0.17$ \\
\hline $\operatorname{ASP}\left({ }^{3} \mathrm{D}_{227}\right)$ & $101.61 \pm 1.76$ & $101.05 \pm 0.50$ & $100.57 \pm 0.81$ \\
\hline \multicolumn{4}{|l|}{ RDS } \\
\hline $\operatorname{IND}\left({ }^{1} \mathrm{D}_{356}\right)$ & $99.88 \pm 1.21$ & $100.38 \pm 0.10$ & $100.21 \pm 0.34$ \\
\hline $\mathrm{BB}\left({ }^{1} \mathrm{D}_{419}\right)$ & $98.91 \pm 0.48$ & $99.78 \pm 0.26$ & $100.67 \pm 0.83$ \\
\hline ACE-K $\left({ }^{1} \mathrm{D}_{248}\right)$ & $98.92 \pm 0.48$ & $100.13 \pm 0.32$ & $99.95 \pm 0.85$ \\
\hline $\operatorname{ASP}\left({ }^{3} \mathrm{D}_{225}\right)$ & $98.54 \pm 1.60$ & $100.45 \pm 0.40$ & $100.39 \pm 0.53$ \\
\hline
\end{tabular}

a The intra-day $(n=5)$, average of three concentrations $\left(2,6,10 \mu \mathrm{gmL}^{-1}\right)$ for IND, BB, ACE-K and ASP repeated three times within the day.

${ }^{\mathrm{b}}$ The inter-day $(n=5)$, average of three concentrations $\left(2,6,10 \mu \mathrm{gmL}^{-1}\right)$ for IND, BB, ACE-K and ASP repeated three times in three successive days.

${ }^{*}$ The values of $\%$ recovery is an average of five determinations of each of five concentration $\left(2-10 \mu \mathrm{gmL}^{-1}\right)$.

RSD\%: Relative standard deviation.

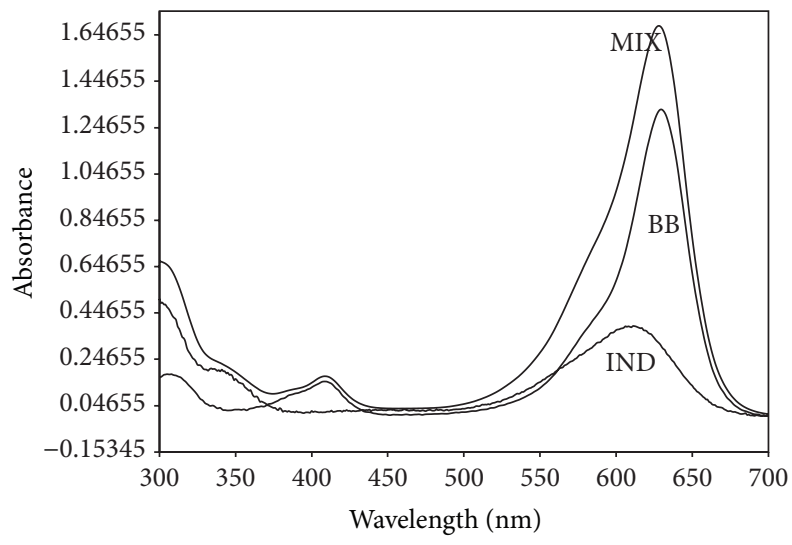

(a)

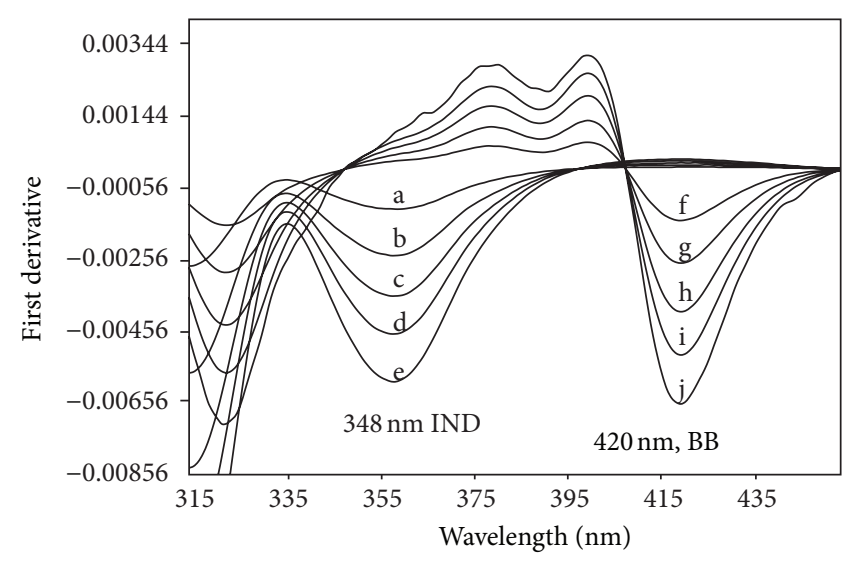

(b)

FIGURE 2: Zero-order spectra (a) of $10 \mu \mathrm{gmL}^{-1} \mathrm{IND}, 10 \mu \mathrm{g} \cdot \mathrm{mL}^{-1} \mathrm{BB}$ and their binary mixture and first derivative spectra (b) of colorants (IND: a: 2, b: 4, c: 6, d: 8 , e: $10 \mu \mathrm{gmL}^{-1}$; BB: f: 2 , g: $4, \mathrm{~h}: 6, \mathrm{i}: 8, \mathrm{j}: 10 \mu \mathrm{gmL}^{-1}$ ). 


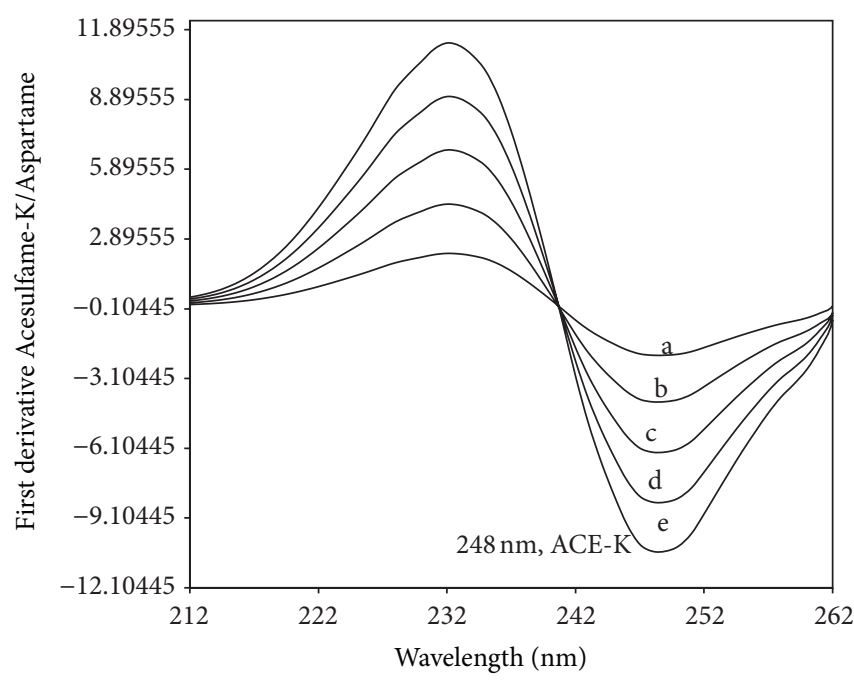

(a)

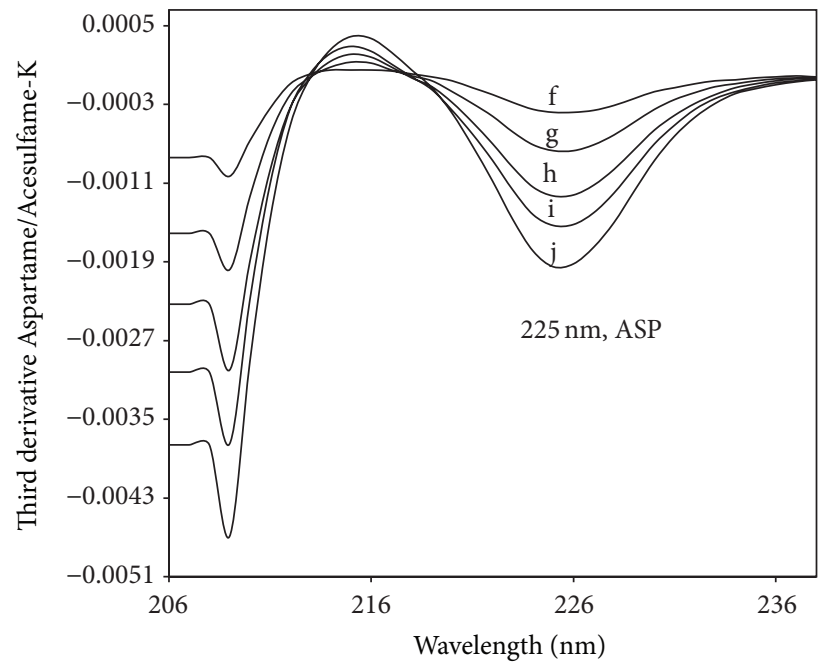

(b)

Figure 3: First derivative of the ratio spectra (a) of ACE-K of a: 2, b: 4, c: 6, d: 8 , e: $10 \mu \mathrm{gmL}^{-1}$, when $6 \mu \mathrm{gmL}^{-1}$ ASP spectrum was used as a divisor, and third derivative of the ratio spectra (b) of ASP of f: $2, \mathrm{~g}: 4, \mathrm{~h}: 6, \mathrm{i}: 8, \mathrm{j}: 10 \mu \mathrm{gmL}^{-1}$, when $4 \mu \mathrm{gmL} \mathrm{ACE}^{-1} \mathrm{~K}$ spectrum was used as a divisor.

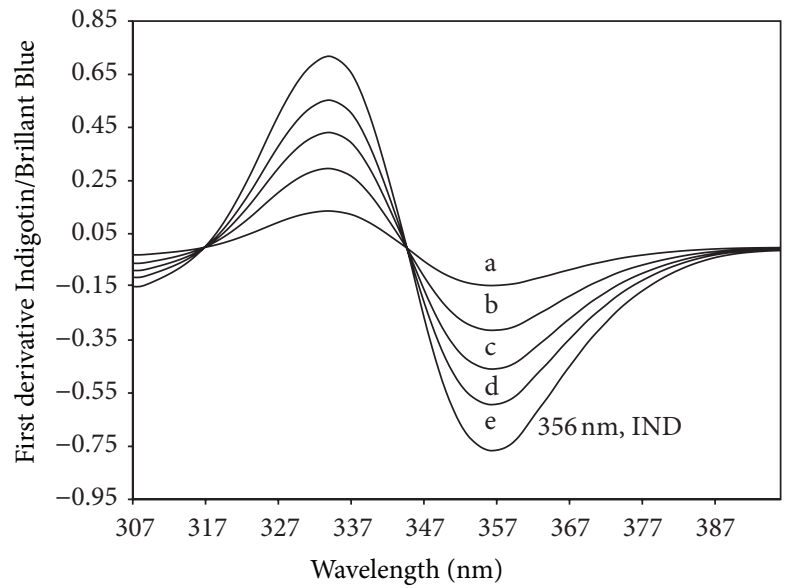

(a)

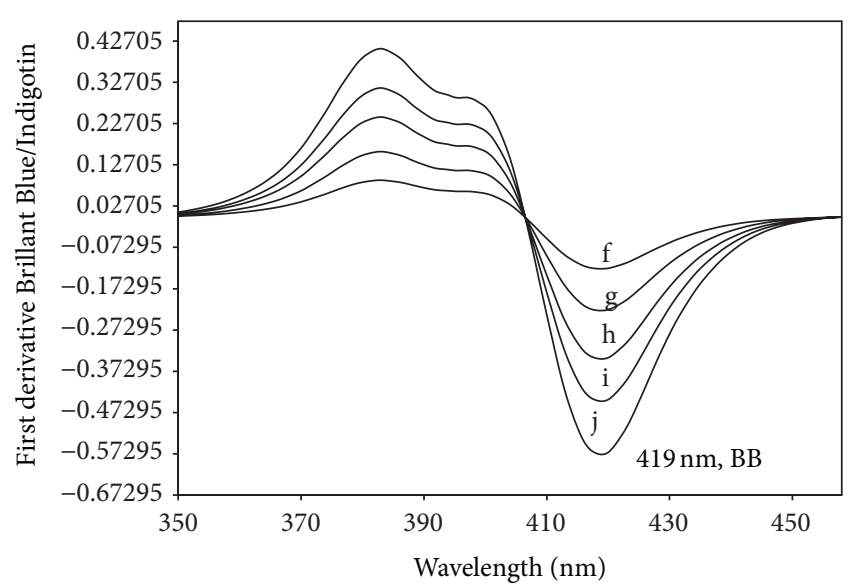

(b)

FIGURE 4: First derivative of the ratio spectra (a) of IND of a: 2, b: 4, c: 6, d: 8, e: $10 \mu \mathrm{gmL}^{-1}$, when $4 \mu \mathrm{gmL}^{-1} \mathrm{BB}$ spectrum was used as a divisor,

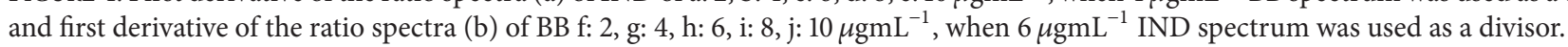

magnetic stirrer for 20 min keeping temperature constant at $40^{\circ} \mathrm{C}$ by a thermostat. After the flask was cooled to the room temperature, centrifuged and volume was completed to $50 \mathrm{~mL}$ with distilled water. The solution was filtered through $0.45 \mu \mathrm{m}$ membrane and transferred into a quartz cell. Absorption spectrum of the solution was recorded between $300-700 \mathrm{~nm}$ against distilled water. Then $0.1 \mathrm{~g}$ activated charcoal was added to decolorize the solution [37], and the mixture was stirred vigorously to adsorb all colorants in the solution. If the solution still has some colour, a small further amount of activated charcoal should be added. The mixture was centrifuged, and $0.5 \mathrm{~mL}$ solution was transferred into $10 \mathrm{~mL}$ volumetric flask. The volume was completed with distilled water, and the absorption spectrum of the solution was recorded between $200-300 \mathrm{~nm}$ against distilled water. The proposed methods were applied to the solutions thus prepared.

(b) Zero-Crossing Derivative Spectrophotometry. The absorption spectra of the standard ACE-K and ASP solutions were recorded between $200-300 \mathrm{~nm}$ against distilled water, using $1.0 \mathrm{~cm}$ quartz cell and stored in a disk file. These spectra were smoothed, and their first- and third-derivative spectra were recorded. The amplitudes, from the baseline to peak at $242 \mathrm{~nm}\left({ }^{1} \mathrm{D}_{242}\right)$, were related to the actual contents of ACE$\mathrm{K}$ and third derivative amplitude at $227 \mathrm{~nm}\left({ }^{3} \mathrm{D}_{227}\right)$ related to ASP content. Absorption spectra of the standard colorant (BB and IND) solutions were recorded within a wavelength 
range of 300-700 $\mathrm{nm}$ against distilled water and stored in a disk file. These spectra were smoothed, and their first derivative spectra were recorded. First-derivative absorbance values were measured at $420 \mathrm{~nm}\left({ }^{1} \mathrm{D}_{420}\right)$ for $\mathrm{BB}$ and $348 \mathrm{~nm}$ $\left({ }^{1} \mathrm{D}_{348}\right)$ for IND.

Laboratory prepared mixtures of ACE-K, ASP, IND, and $\mathrm{BB}$ with different ratios in the concentration range stated in Table 2 were prepared. These solutions and commercial product were analyzed as described above.

(c) Ratio Derivative Spectrophotometry. According to the theory of the ratio-spectra derivative method, absorption spectra of the standard ACE-K solutions were recorded with $1 \mathrm{~nm}$ resolution in the range $200-300 \mathrm{~nm}$ against distilled water and stored in the computer. The stored spectra of the ACE$\mathrm{K}$ were divided, wavelength by wavelength, by a standard spectrum of $6 \mu \mathrm{g} \mathrm{mL}^{-1}$ ASP solution. The ratio spectra were smoothed, and their first-derivative spectra were recorded. In the binary mixtures, we can determine the amount of ACE-K by measuring the amplitudes at $248 \mathrm{~nm}\left({ }^{1} \mathrm{D}_{248}\right)$ corresponding to a minimum. On the other hand, stored spectra of ASP solutions were divided by a standard spectrum of $4 \mu \mathrm{g} \mathrm{mL}^{-1} \mathrm{ACE}-\mathrm{K}$ solution. In the same way as we have previously described, we obtained third derivative spectra from smoothed ratio spectra. Now, ASP can be determined by measuring the signals at $225 \mathrm{~nm}\left({ }^{3} \mathrm{D}_{225}\right)$ corresponding to a minimum wavelength.

Absorption spectra of the solutions at different concentrations of $\mathrm{BB}$ were recorded and divided by a standard spectrum of $6.0 \mu \mathrm{gmL}^{-1} \mathrm{IND}$, and the ratio spectra were obtained. All spectra were stored in computer. Then the first derivative spectra of the ratio spectra were plotted. In the binary mixture, the amount of $\mathrm{BB}$ can be determined by measuring the first derivative signal at $419 \mathrm{~nm}\left({ }^{1} \mathrm{D}_{419}\right)$ corresponding to a minimum in the spectral region $300-450 \mathrm{~nm}$. In the same way, the absorption spectra of the solution at different concentration of IND were divided by the standard spectrum of $4.0 \mu \mathrm{gmL}^{-1} \mathrm{BB}$ solution. First derivative spectra of the ratio spectra were plotted from the obtained ratio spectra. The concentration of IND was determined by measuring the signal at $356 \mathrm{~nm}\left({ }^{1} \mathrm{D}_{356}\right)$ corresponding to a minimum in the first derivative of the ratio spectra in $300-400 \mathrm{~nm}$ region. Laboratory-prepared mixtures at different ratios were prepared in the concentration range stated in Table 2, and then the ratio derivative absorbance values were measured at the selected wavelengths mentioned in Table 1.

Commercial product was also analyzed according to the procedure described above.

\section{Result and Discussion}

3.1. Zero-Crossing Derivative Spectrophotometry. Zero-order absorption spectra of ACE, ASP and, their mixture in the 200-300 nm wavelength region are shown in Figure 1(a). The extensive overlap of the spectral bands of the two sweeteners prevents the use of conventional UV spectrophotometry for assaying binary mixtures. In contrast to zero-order spectra, derivative spectra show more resolution in terms of zero-crossing points shown in Figures 1(b) and 1(c). Firstderivative wavelength was considered $242 \mathrm{~nm}\left({ }^{1} \mathrm{D}_{242}\right)$ for the determination of ACE-K, and the third derivative wavelength $227 \mathrm{~nm}\left({ }^{3} \mathrm{D}_{227}\right)$ was considered for the determination of ASP. At $242 \mathrm{~nm}\left({ }^{1} \mathrm{D}_{242}\right)$ ASP shows zero absorbance; therefore ACE- $K$ is determined at this wavelength without any interference of ASP. At $227 \mathrm{~nm}\left({ }^{3} \mathrm{D}_{227}\right)$ there is no contribution of ACE-K and ASP is determined at this wavelength in the presence of ACE-K represented in Figure 1(c).

Absorption spectra of $\mathrm{BB}, \mathrm{IND}$, and their mixture were recorded within a wavelength range of $300-700 \mathrm{~nm}$ are shown in Figure 2(a). As can be seen, the spectra of the colorants are strongly overlapped in the 400-600 nm wavelength region. Hence, direct absorption measurement for assaying binary mixture seems to be impossible. On the other hand, the absorption spectra of the two components within a wavelength range $300-450 \mathrm{~nm}$ were less overlapped than the region $450-700 \mathrm{~nm}$. So, in this work, the region of 300$450 \mathrm{~nm}$ was chosen to analyze each colorant. Figure 2(b) shows the first derivative spectra of IND and BB, and these spectra allow the simultaneous determination of colorants. ${ }^{1} \mathrm{D}$ spectrum of IND shows a well-defined minimum at $348 \mathrm{~nm}\left({ }^{1} \mathrm{D}_{348}\right)$, while $\mathrm{BB}$ has a zero ${ }^{1} \mathrm{D}$ value at the same wavelength. $\mathrm{BB}$ has a minimum ${ }^{1} \mathrm{D}$ value at $420 \mathrm{~nm}\left({ }^{1} \mathrm{D}_{420}\right)$ which IND exhibits no contribution. Therefore, at these selected wavelengths, two colorants can be quantified in the presence of each other without interference.

For the quantitative analysis of colorants and sweeteners, the representative linear equations and other analytical parameters are shown in Table 1. The correlation coefficients for active compounds showed good linearity in the concentration range pronounced in the previous section.

3.2. Ratio Derivative Spectrophotometry. Ratio Derivative Spectrophotometry permits determination of a component in their mixture at the wavelengths corresponding to a maximum or a minimum and, also, the use of the peak to peak between consecutive maximum and minimum. The values of absorbance in these points permit one, in some cases, to reach a better sensitivity. The main instrumental conditions were optimized to obtain the most distinct curve of first and third derivative of the ratio spectra. Divisor concentration is the main instrumental parameter. The ratio spectra derivative method permits the use of the different concentrations as the divisor to obtain the different calibration graphs. In a preliminary investigation, for selecting the standard solution as a divisor at an appropriate concentration, concentrations of $\mathrm{ASP}$ and ACE-K in the range $2-10 \mu \mathrm{gmL}^{-1}$ were tested. The concentration of $4 \mu \mathrm{gmL}^{-1}$ of ACE-K and $6 \mu \mathrm{gmL}^{-1}$ of ASP as a divisor gave the best results in terms of signal to noise ratio and highest correlation coefficient values, being an indication of the quality of fitting of the data to the straight line. Figure 3(a) shows the first derivative of the ratio-spectra of different amounts of ACE-K solutions (divided by a standard spectrum of $6 \mu \mathrm{gmL}^{-1}$ ASP solution). The first derivative amplitude at $248 \mathrm{~nm}\left({ }^{1} \mathrm{D}_{248}\right)$ corresponding to a minimum was proportional to ACE-K concentration. Figure 3(b) shows the third derivative ratio spectra of different concentration 
of ASP standard solutions using the spectrum of $4 \mu \mathrm{gmL}^{-1}$ ACE-K solution as a divisor. The concentration of ASP is proportional to the amplitude of the minimum at $225 \mathrm{~nm}$ $\left({ }^{3} \mathrm{D}_{225}\right)$.

As can be seen in Figure 2(a), determination of IND and $\mathrm{BB}$ was not possible by direct measurements of absorbance in zero-order spectra. On the other hand, derivative spectroscopy shows more resolution and makes it possible to analyze each colorant in the presence of one another as well as in the presence of other ingredients without any pretreatment. Figure 4(a) indicates first derivative of the ratio spectra of IND at increasing concentrations values, in the solution of distilled water, which were obtained by dividing each of absorption spectrum with the spectrum of the standard solution of $\mathrm{BB}$. The calibration graph of IND was established by measuring the signal at $356 \mathrm{~nm}\left({ }^{1} \mathrm{D}_{356}\right)$ corresponding to a minimum (Table 1). Also Figure 4(b) indicates the first derivative of the ratio spectra of different BB standards (spectra divided by the spectrum of $6 \mu \mathrm{gmL}^{-1}$ $\mathrm{BB})$.

Moreover, each set of standard solution of tested colorants and sweeteners, within the concentration range stated in Table 1, was assayed by the proposed ratio-spectra derivative method. The regression line obtained with one divisor concentration with their statistical data compiled in Table 1. The correlation coefficients were (from 0.9992 to 0.9999 ) indicating good linearity, and very small intercepts values were obtained. For the determination of colorants and sweeteners in their synthetic mixtures and in the chewing-gum sample, only the calibration graphs, that were obtained by measuring the signals at $356 \mathrm{~nm}\left({ }^{1} \mathrm{D}\right)$ for IND; $419 \mathrm{~nm}\left({ }^{1} \mathrm{D}_{419}\right)$ for BB and $248 \mathrm{~nm}\left({ }^{1} \mathrm{D}_{248}\right)$ for ACE-K; $225 \mathrm{~nm}\left({ }^{3} \mathrm{D}_{225}\right)$ for ASP in the first and third derivative of the spectra, were used.

The developed methods were validated, and accuracy is shown in Table 2. Summary of various validation parameters is given listed in Tables 1 and 2, and the results of chewinggum analysis are listed in Table 3 . A good coincidence was observed for the assay results of the chewing-gum sample by application of two methods in this paper (Table 3).

3.3. Validation of the Methods. The methods were validated as regards in selectivity, linearity, precision (within and between days) limit of detection (LOD), and limit of quantification (LOQ). The validation results are shown in Tables 1 and 2.

3.3.1. Linearity. Linearity was studied for each colorant and sweetener in the concentration ranged stated in Table 1 . The regression equations for the results were derived using the least-squares method. In all cases, Beer's law plots $(n: 5)$ were linear with very small intercepts and good correlation coefficients (from 0.9992 -to 0.9999). The results of the linearity study for all colorants and sweeteners with two methods are given in Table 1.

3.3.2. Precision, Accuracy and Percent Recoveries. To test the repeatability of the proposed methods, five separate determinations at different concentration levels were carried out for each colorant in the presence of certain concentration of the other component and for each sweetener in the presence of other component. The percentage recoveries of each colorant and sweetener were calculated by comparing the found and added concentration ( $\mu \mathrm{g}$ found $/ \mu \mathrm{g}$ added) $\times$ 100. Mean recoveries are given in Table 2. No interference occurred in the presence of the others, and the mean percentage recoveries were found in the range $99.50 \%-103.5 \%$ for derivative zero-crossing and for derivative ratio method $98.00 \%-103.5 \%$. Recovery values were close to $100 \%$, and they indicate that the developed methods were appropriate for accurate determination of sweeteners and colorants in their quaternary mixtures. Beside these, RSD was calculated as $<1.76 \%$ for the determination of BB, IND, ACE-K, and ASP, and these values indicate that the developed methods could estimate concentration values with a good precision. To evaluate the intra-day precision and inter-day precision (as RSD), each sample was analyzed three times in the same day. Three replicates of each sample preparation and analysis were done to evaluate inter-day precision (as RSD) in three different days. The concentration values for both intra-day precision and inter-day precision were calculated. Finally, the mean of RSD\% (RSD\%: $(S / \bar{X}) \times 100$, where $S$ is the standard deviation and $\bar{X}$ is the mean of the sample analyzed) was taken for conclusion. The results are shown in Table 2. These results show the accuracy and reproducibility of the assay. Thus, it was concluded that there was no significant difference for the assay, which was tested within the day and between the days. The results obtained show that the relative standard deviation values were less than $2 \%$ which indicate high degree of precision of the proposed methods. The low values of standard deviations showed that the methods were precise.

3.3.3. Limit of Detection (LOD) and Limit of Quantification (LOQ). LOD and LOQ were calculated using the following equations:

$$
\begin{gathered}
\text { LOD : } \bar{X}_{b_{1}}+3 S_{b_{1}}, \\
\text { LOQ : } \overline{X_{b_{1}}}+10 S_{b_{1}},
\end{gathered}
$$

where $\overline{X_{b_{1}}}$ is the mean concentration of the blank and $S_{b_{1}}$ is the standard deviation of the blank. Blank was measured 5-fold on spectrophotometer. LOD is expressed as the analyte concentration corresponding to the sample blank value plus three standard deviations, and LOQ is the analyte concentration corresponding to the sample blank value ten plus standard deviations. The data are presented in Table 1 . The LOD and the LOQ values of the two studied colorants ranged from 0.14 to $0.33 \mu \mathrm{gmL}^{-1}$ and from 0.22 to $0.47 \mu \mathrm{gmL}^{-1}$, respectively. The LOD and the LOQ values of the two studied sweeteners ranged from 0.04 to 0.07 and from 0.06 to $0.38 \mu \mathrm{gmL}^{-1}$, respectively. Nevertheless, in general, the method that used the derivative ratio spectra shows better limit detection and quantification.

3.4. Dosage Forms Analysis. The present methods were applied for the simultaneous determination of the above 
TABLE 3: Assay results for the determination of IND, BB, ACE and ASP in a commercial chewing-gum sample by proposed methods.

\begin{tabular}{lccc}
\hline Methods & Analyte & Selected Wavelength $(\mathrm{nm})$ & Assay results $\left(\mathrm{mgKg}^{-1}\right)(\mathrm{mean} \pm \mathrm{SD})^{*}\left(t_{\text {calculated }} ; F_{\text {calculated }}\right)^{* *}$ \\
\hline \multirow{4}{*}{ ZCDS } & IND & ${ }^{1} \mathrm{D}_{348}$ & $14.60 \pm 0.82(0.26 ; 1.26)$ \\
& $\mathrm{BB}$ & ${ }^{1} \mathrm{D}_{420}$ & $4.66 \pm 0.89(0.84 ; 1.02)$ \\
& $\mathrm{ACE}-\mathrm{K}$ & ${ }^{1} \mathrm{D}_{242}$ & $530.81 \pm 0.79(1.42 ; 1.92)$ \\
& $\mathrm{3} P \mathrm{~B} \mathrm{D}_{227}$ & $458.66 \pm 0.98(1.76 ; 1.81)$ \\
\multirow{2}{*}{ RDS } & $\mathrm{IND}$ & ${ }^{1} \mathrm{D}_{356}$ & $14.73 \pm 0.73$ \\
& $\mathrm{BB}$ & ${ }^{1} \mathrm{D}_{419}$ & $4.18 \pm 0.90$ \\
& $\mathrm{ACE}-\mathrm{K}$ & ${ }^{1} \mathrm{D}_{248}$ & $531.43 \pm 0.57$ \\
& $\mathrm{ASP}$ & ${ }^{3} \mathrm{D}_{225}$ & $457.36 \pm 1.32$ \\
\hline
\end{tabular}

${ }^{*}$ Results obtained are the average of five experiments for each method; SD: Standard deviation.

${ }^{* *}$ The corresponding theoretical value for $t$ and $F$ at $P: 0.05$ ( $\left.t_{\text {therorictal }}: 2.31 ; F_{\text {therorictal }}: 6.39\right)$.

mentioned quaternary mixture in their available commercial chewing-gum sample. The assay results obtained by developed methods were much closed to each other and low values of RSD\%, suggesting that the developed methods have high precision. The results obtained by applying the ZCDS method were statistically compared with those obtained by the RDS method. The values of calculated $t$ - and $F$ - are less than the tabulated ones, which reveals that there is no significant difference with respect to accuracy and precision between the proposed methods. The summary of these results is given in Table 3. The developed two spectrophotometric methods showed the advantages of being simple, rapid, cost effective, and time saving and can be employed as an alternative analytical method for the simultaneous determination of these two colorants and two sweeteners. Therefore, the methods are suggested for routine analysis of BB-IND and ACE-K-ASP in chewing-gum or the other food samples.

\section{Conclusions}

Two analytical spectrophotometric methods were developed and validated thoroughly for the quantitative determination of ACE-K, ASP, IND, and BB in synthetic mixtures and chewing gum sample. This paper demonstrates the potential of derivative (first and third) spectrophotometry and ratio derivative (first and third) spectrophotometry as an analytical technique and its usefulness to accurately, rapidly, simply, and simultaneously quantify four active compounds in commercially chewing-gum samples. By the ratio spectra first-and third-order derivative method, we can measure the absorbances of the sweeteners and colorants at no critical wavelengths corresponding to a maximum or a minimum and also use the addition of absorbance values in consecutive maximum and minimum (peak to peak). This method is very useful because in some cases it permits one to achieve a better sensitivity. Expensive methods such as HPLC or electrophoresis seem unnecessary for the determination of these substances in foods since their concentration in foods and drinks are not as low as the one in blood, pharmaceuticals, and cosmetics. The short analysis time, rapid, low costs, and environmental protection are the other advantages of proposed methods compared to HPLC or other existing methods. The proposed methods were completely validated and suitable for quality control laboratories, where economy and time are essential.

\section{Acknowledgment}

This research has been supported by scientific research project coordination center of Y Y ld $\imath z$ Technical University (project number: 2011-01-02-GEP12).

\section{References}

[1] E. C. Vidotti, W. F. Costa, and C. C. Oliveira, "Development of a green chromatographic method for determination of colorants in food samples," Talanta, vol. 68, no. 3, pp. 516-521, 2006.

[2] G. J. Kapadia, H. Tokuda, R. Sridhar et al., "Cancer chemopreventive activity of synthetic colorants used in foods, pharmaceuticals and cosmetic preparations," Cancer Letters, vol. 129, no. 1, pp. 87-95, 1998.

[3] V. Rus, C. Gherman, V. Miclǎuş, A. Mihalca, and G. C. Nadǎş, "Comparative toxicity of food dyes on liver and kidney in guinea pigs: a histopathological study," Annals of the Romanian Society for Cell Biology, vol. 15, no. 1, pp. 161-165, 2010.

[4] E. C. Vidotti, J. C. Cancino, C. C. Oliveira, and M. D. C. E. Rollemberg, "Simultaneous determination of food dyes by first derivative spectrophotometry with sorption onto polyurethane foam," Analytical Sciences, vol. 21, no. 2, pp. 149-153, 2005.

[5] N. Pourreza and S. Elhami, "Cloud point extraction and spectrophotometric determination of amaranth in food samples using nonionic surfactant Triton X-100 and tetrabutylammonium hydrogen sulfate," Journal of the Iranian Chemical Society, vol. 6, no. 4, pp. 784-788, 2009.

[6] N. Pourreza and M. Ghomi, "Simultaneous cloud point extraction and spectrophotometric determination of carmoisine and brilliant blue FCF in food samples," Talanta, vol. 84, no. 1, pp. 240-243, 2011.

[7] R. Li, Z.-T. Jiang, and R.-X. Wang, "Solid phase extraction combined direct spectrophotometric determination of brilliant blue in food using $\beta$-cyclodextrin polymer," Food Analytical Methods, vol. 2, no. 4, pp. 264-270, 2009.

[8] A. S. Nateri and E. Ekrami, "Quantitative analysis of bicomponent dye solutions by derivative spectrophotometry," Pigment and Resin Technology, vol. 38, no. 1, pp. 43-48, 2009.

[9] M. Kucharska and J. Grabka, "A review of chromatographic methods for determination of synthetic food dyes," Talanta, vol. 80, no. 3, pp. 1045-1051, 2010. 
[10] N. Yoshioka and K. Ichihashi, "Determination of 40 synthetic food colors in drinks and candies by high-performance liquid chromatography using a short column with photodiode array detection," Talanta, vol. 74, no. 5, pp. 1408-1413, 2008.

[11] K. S. Minioti, C. F. Sakellariou, and N. S. Thomaidis, "Determination of 13 synthetic food colorants in water-soluble foods by reversed-phase high-performance liquid chromatography coupled with diode-array detector," Analytica Chimica Acta, vol. 583, no. 1, pp. 103-110, 2007.

[12] N. Vachirapatama, J. Mahajaroensiri, and W. Visessanguan, "Identification and determination of seven synthetic dyes in foodstuffs and soft drinks on monolithic C18 column by high performance liquid chromatography," Journal of Food and Drug Analysis, vol. 16, no. 5, pp. 77-82, 2008.

[13] M. C. Boyce, "Determination of additives in food by capillary electrophoresis," Electrophoresis, vol. 22, no. 8, pp. 1447-1459, 2001.

[14] S. Chanlon, L. Joly-Pottuz, M. Chatelut, O. Vittori, and J. L. Cretier, "Determination of carmoisine, allura red and ponceau $4 \mathrm{R}$ in sweets and soft drinks by differential pulse polarography," Journal of Food Composition and Analysis, vol. 18, no. 6, pp. 503515, 2005.

[15] A. H. Alghamdi, H. M. Alshammery, M. A. Abdalla, and A. F. Alghamdi, "Determination of carmine food dye (E120) in foodstuffs by stripping voltammetry," Journal of AOAC International, vol. 92, no. 5, pp. 1454-1459, 2009.

[16] N. E. Llamas, M. Garrido, M. S. D. Nezio, and B. S. F. Band, "Second order advantage in the determination of amaranth, sunset yellow FCF and tartrazine by UV-vis and multivariate curve resolution-alternating least squares," Analytica Chimica Acta, vol. 655, no. 1-2, pp. 38-42, 2009.

[17] D. W. Lachenmeier and W. Kessler, "Multivariate curve resolution of spectrophotometric data for the determination of artificial food colors," Journal of Agricultural and Food Chemistry, vol. 56, no. 14, pp. 5463-5468, 2008.

[18] Y. S. Al-Degsa, A. H. El-Sheikha, M. A. Al-Ghoutib, and M. S. Sunjuka, "Determination of commercial colorants in different water bodies using partial least squares regression (PLS) A Chemometric Study," Jordan Journal of Chemistry, vol. 3, no. 3, pp. 321-336, 2008.

[19] Y. Ni, Y. Wang, and S. Kokot, "Simultaneous kinetic spectrophotometric analysis of five synthetic food colorants with the aid of chemometrics," Talanta, vol. 78, no. 2, pp. 432-441, 2009.

[20] C. R. Whitehouse, J. Boullata, and L. A. McCauley, "The potential toxicity of artificial sweeteners," AAOHN Journal, vol. 56, no. 6, pp. 251-261, 2008.

[21] A. Wasik, J. McCourt, and M. Buchgraber, "Simultaneous determination of nine intense sweeteners in foodstuffs by high performance liquid chromatography and evaporative light scattering detection-Development and single-laboratory validation," Journal of Chromatography A, vol. 1157, no. 1-2, pp. 187196, 2007.

[22] D. J. Yang and B. Chen, "Simultaneous determination of nonnutritive sweeteners in foods by HPLC/ESI-MS," Journal of Agricultural and Food Chemistry, vol. 57, no. 8, pp. 3022-3027, 2009.

[23] M. Serdar and Z. Knežević, "Determination of artificial sweeteners in beverages and special nutritional products using high performance liquid chromatography," Arhiv za Higijenu Rada $i$ Toksikologiju, vol. 62, no. 2, pp. 169-173, 2011.

[24] A. Zygler, A. Wasik, A. Kot-Wasik, and J. Namieśnik, "Determination of nine high-intensity sweeteners in various foods by high-performance liquid chromatography with mass spectrometric detection," Analytical and Bioanalytical Chemistry, vol. 400, no. 7, pp. 2159-2172, 2011.

[25] Y. Zhu, Y. Guo, M. Ye, and F. S. James, "Separation and simultaneous determination of four artificial sweeteners in food and beverages by ion chromatography," Journal of Chromatography A, vol. 1085, no. 1, pp. 143-146, 2005.

[26] M. Herrmannová, L. Křivánková, M. Bartoš, and K. Vytřas, "Direct simultaneous determination of eight sweeteners in foods by capillary isotachophoresis," Journal of Separation Science, vol. 29, no. 8, pp. 1132-1137, 2006.

[27] S. Armenta, S. Garrigues, and M. De La Guardia, "Sweeteners determination in table top formulations using FT-Raman spectrometry and chemometric analysis," Analytica Chimica Acta, vol. 521, no. 2, pp. 149-155, 2004.

[28] M. A. Cantarelli, R. G. Pellerano, E. J. Marchevsky, and J. M. Camiña, "Simultaneous determination of saccharin and aspartame in commercial noncaloric sweeteners using the PLS2 multivariate calibration method and validation by capillary electrophoresis," Journal of Agricultural and Food Chemistry, vol. 56, no. 20, pp. 9345-9349, 2008.

[29] Y. Ni, W. Xiao, and S. Kokot, "A differential kinetic spectrophotometric method for determination of three sulphanilamide artificial sweeteners with the aid of chemometrics," Food Chemistry, vol. 113, no. 4, pp. 1339-1345, 2009.

[30] N. E. Llamas, M. S. Di Nezio, and M. E. Palomeque, "Direct determination of saccharin and acesulfame-K in sweeteners and fruit juices powders," Food Analytical Methods, vol. 1, no. 1, pp. 43-48, 2008.

[31] A. Zygler, A. Wasik, and J. Namieśnik, "Analytical methodologies for determination of artificial sweeteners in foodstuffs," Trends in Analytical Chemistry, vol. 28, no. 9, pp. 1082-1102, 2009.

[32] Z. Şentürk, N. Erk, S. A. Özkan, C. Akay, and S. Cevheroglu, "Analysis of theophylline and ephedrine hydrochloride in tablets by ratio-spectra derivative spectrophotometry and LC," Journal of Pharmaceutical and Biomedical Analysis, vol. 29, no. 1-2, pp. 291-298, 2002.

[33] F. Salinas, J. J. B. Nevado, and A. E. Mansilla, "A new spectrophotometric method for quantitative multicomponent analysis resolution of mixtures of salicylic and salicyluric acids," Talanta, vol. 37, no. 3, pp. 347-351, 1990.

[34] S. Saraf, "Various UV spectrophotometric simultaneous estimation methods," Pharmainfo.net. Latest Reviews, vol. 4, no. 2, 2006.

[35] H. N. Dave, R. C. Mashru, and A. R. Thakkar, "Simultaneous determination of salbutamol sulphate, bromhexine hydrochloride and etofylline in pharmaceutical formulations with the use of four rapid derivative spectrophotometric methods," Analytica Chimica Acta, vol. 597, no. 1, pp. 113-120, 2007.

[36] M. I. Walash, M. S. Rizk, Z. A. Sheribah, and M. M. Salim, "Derivative spectrophotometric analysis of benzophenone (as an impurity) in phenytoin," Chemistry Central Journal, vol. 5, no. 1, pp. 85-95, 2011.

[37] Megazyme International Ireland Ltd, "Aspartame assay procedure 01/05," 2004, http://www.megazyme.com. 

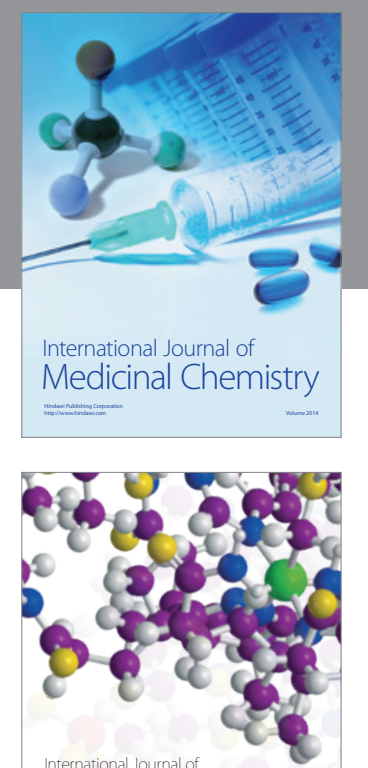

\section{Carbohydrate} Chemistry

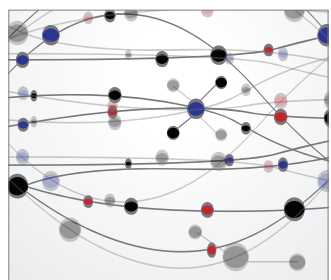

The Scientific World Journal
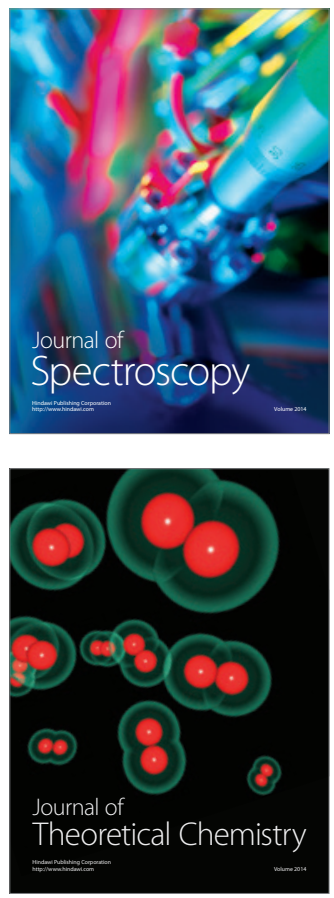
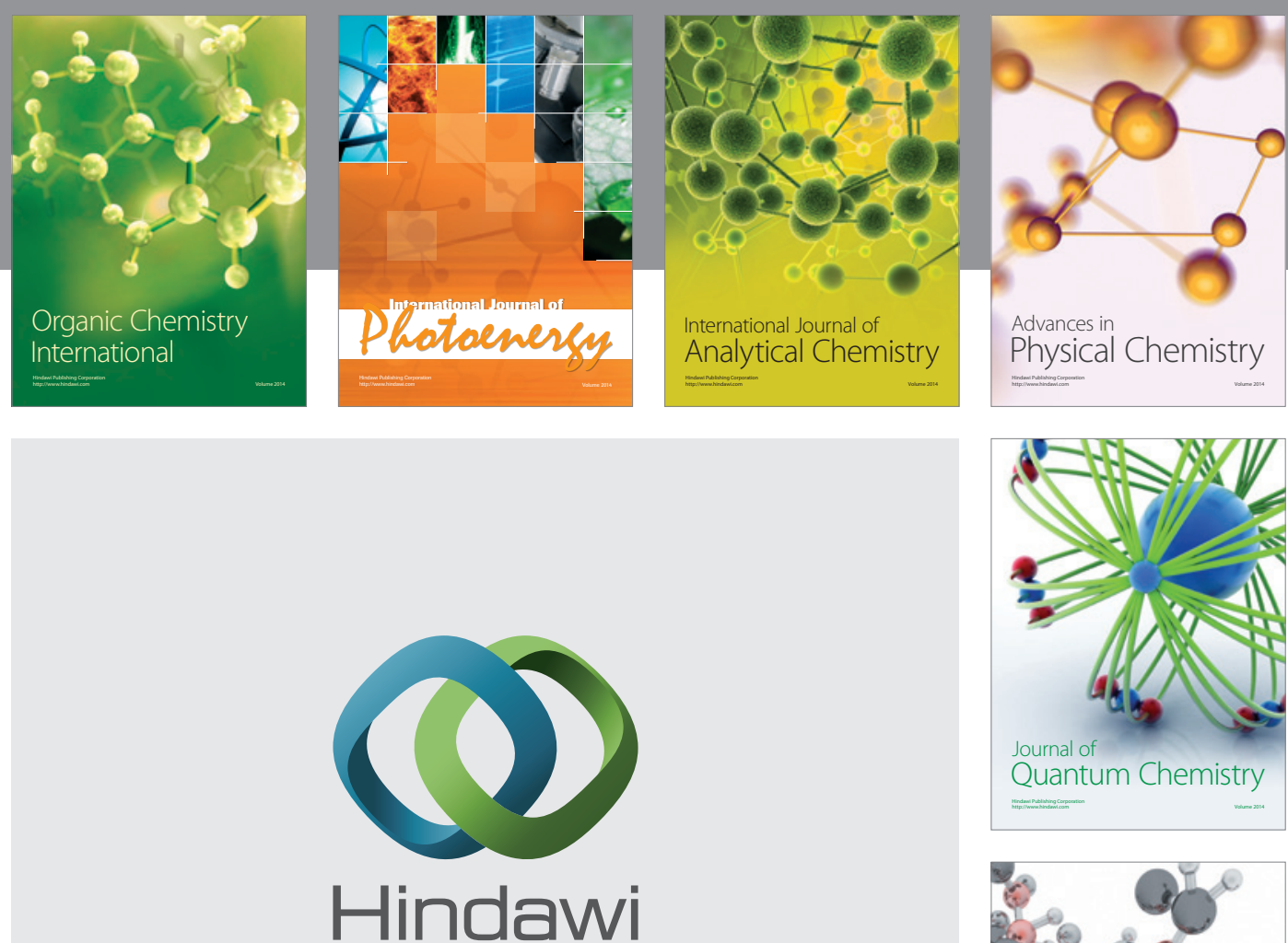

Submit your manuscripts at

http://www.hindawi.com

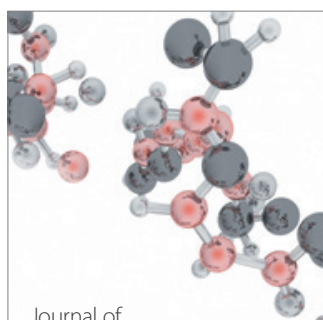

Analytical Methods

in Chemistry

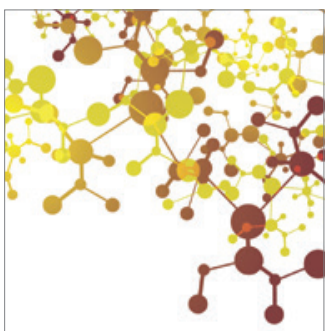

Journal of

Applied Chemistry

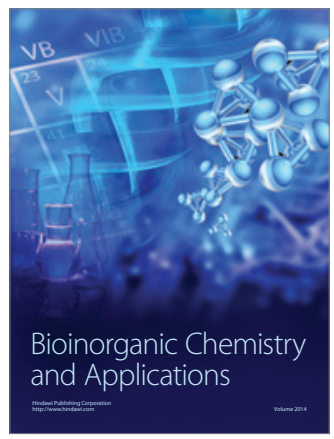

Inorganic Chemistry
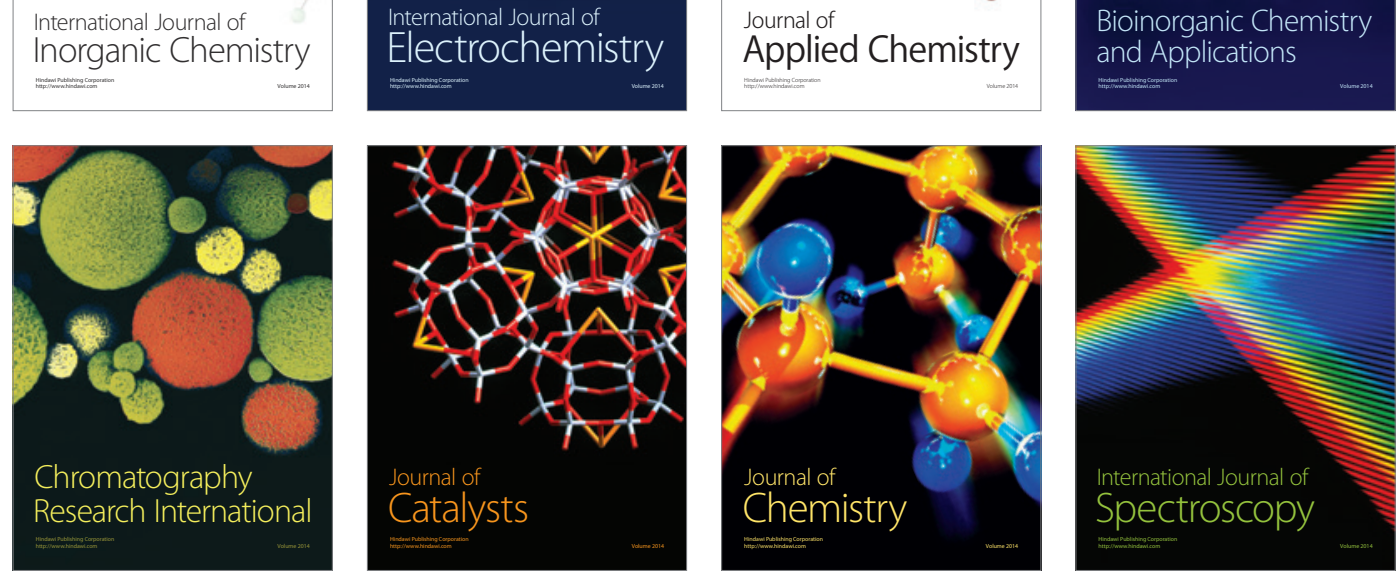